\title{
Diferencia esperada de progenie como herramienta de selección para peso al destete en ganado Brahman
}

\author{
Expected progeny difference as a tool for selection for weaning \\ weight in Brahman cattle
}

\author{
Donicer Montes , $^{1 *}$ Esp, Oscar Vergara G, ${ }^{2}$ Ph.D, Wilson Barragán, ${ }^{3}$ Zoot.
}

\begin{abstract}
${ }^{1}$ Universidad de Sucre, Facultad de Ciencias Agropecuarias, Departamento de Zootecnia, Sincelejo, Colombia. ${ }^{2}$ Universidad de Córdoba, Facultad de Medicina Veterinaria y Zootecnia. Departamento de Ciencias Pecuarias. Montería, Colombia. ${ }^{3}$ Ejercicio Particular. Grupo Reproducción y Mejoramiento Genético Animal *Correspondencia: donicermontes@hotmail.com
\end{abstract}

Recibido: Noviembre de 2009; Aceptado: Julio de 2010.

\section{RESUMEN}

Objetivo. Determinar la diferencia esperada de progenie (DEP's) para el peso al destete (PD) en ganado Brahman como criterio de selección de futuros reproductores. Materiales y métodos. Se utilizaron 552 registros (PD) recolectados entre 1997 y 2007 de la hacienda Mundo Nuevo (San Onofre, Sucre, Colombia), provenientes de 33 padres. El modelo estadístico utilizado incluyó los efectos fijos de año y época de nacimiento, sexo del ternero, número de partos de la vaca y el efecto aleatorio del padre. Para el análisis, se utilizó el método de los cuadrados mínimos mediante el procedimiento PROC GLM, contenido en el statistical analysis system (SAS). La heredabilidad fue estimada con los componentes de varianza entre y dentro de toro, obtenidos por el procedimiento PROC VARCOMP. Las diferencias esperadas de progenies (DEP's), fueron calculadas a través del método de mejor predictor lineal insesgado (BLUP). Resultados. La media del PD fue $238.5 \pm 31.6 \mathrm{~kg}$. Los efectos de padre, sexo, número de partos y año de nacimiento fueron significativos $(p \leq 0.05)$. La heredabilidad para PD fue $0.43 \pm 0.15$. Las DEP's variaron entre -16.9 $y+17 \mathrm{~kg}$, con una exactitud (EXA) que osciló entre 0.71 y 0.78 . Conclusiones. La significancia de las diferentes fuentes de variación de PD coincide con la mayoría de los reportes referenciados en la literatura. El valor estimado de la heredabilidad para PD, indica que se puede lograr progreso genético para PD. Los resultados de las (DEP's) evidenciaron que el $52 \%$ de los toros aportan variaciones negativas para PD.

Palabras clave: Fenotipos, heredabilidad, progenie, peso al destete. (Fuente: AIMS) 


\section{ABSTRACT}

Objective. To determine the Expected Progeny Differences (EPD's) for the weaning weight of the Brahman cattle as a criterion for selection of future sires. Materials and methods. 552 records of weaning weight (WW) of 33 sires belonging to the farm Mundo Nuevo, San Onofre, Sucre, Colombia, were recorded during 1997 to 2007 . The statistical model included the fixed effects: year of birth, season weaning, sex of calf, the number of calving and the random effect of the sire. For the analysis of the environmental factors the method of least squares was used, through of the GLM procedure, contained in the Statistical Analysis System. The heritability was obtained through the variance components using the Varcomp procedure. The EPD's were calculated using the method Best Linear Unbiased Prediction (BLUP). Results. The average for WW was $238.5 \pm 31.6 \mathrm{~kg}$. Sire, sex, number of calving, and year of birth were significant $(p \leq 0.05)$. Estimated heritability was $0.43 \pm 0.15$. EPD's ranged between -16.9 and $+17 \mathrm{~kg}$. Accuracies (ACC) ranged from 0.71 at 0.78 . Conclusions. The significance of different sources of variation of WW showed no differences with most of the literature consulted. The estimated heritability indicates that genetic progress can be achieved for WW. The results of the EPD's showed that $52 \%$ of the sires showed negative variations for WW.

Key words: Heritability, phenotype, progeny, weaning weight. (Source: AIMS)

\section{INTRODUCCIÓN}

La ganadería colombiana está caracterizada por ser una actividad extractiva, con bajos niveles de inversión y un deficiente desarrollo de acciones administrativas que la promuevan empresarialmente en un mercado globalizado, que es altamente competitivo (1). Las explotaciones ganaderas bovinas en las Sabanas de Sucre y Córdoba basan su actividad técnica en el mejoramiento de potreros, nutrición, salud y administración, descuidando otros aspectos igualmente importantes. En muchos hatos ganaderos, el progreso genético de características de importancia económica no es posible que se determine, debido a la falta de información registrada de los animales. Este proceso no se ejecuta por la manifiesta apatía de los ganaderos en aplicar este tipo de procedimiento en el manejo del hato, quien lo ve como un gasto y no como una inversión (2).

En la últimas décadas, con el desarrollo de la informática y de métodos estadísticos es posible predecir el valor genético de los animales, obteniéndose lo que en ganado de carne se conoce como Diferencias Esperadas Entre Progenie (DEP's) (3), evaluación que se describe como la diferencia que se espera que la progenie de un determinado individuo produzca en promedio, con relación al promedio de la progenie de otro individuo o base genética utilizada. Este valor va acompañado de un valor de confiabilidad, el cual indica en qué medida el valor de DEP's se va a cumplir y reflejar en la progenie (4). La determinación de los DEP's, permiten seleccionar los animales genéticamente superiores $y$ contribuye a aumentar el rendimiento productivo y económico del hato. Por lo anterior y aprovechando la disponibilidad de una base de datos de la hacienda Mundo Nuevo del municipio de San Onofre - Sucre Colombia, se consideró como objetivo del presente estudio predecir las DEP's para el peso al destete de 33 reproductores de la raza Brahman y utilizar esta información como criterio de selección.

\section{MATERIALES Y MÉTODOS}

Sitio de Estudio. La información utilizada pertenece a la hacienda Mundo Nuevo, ubicada en el corregimiento del Pueblito, municipio de San Onofre (Sucre-Colombia). Ecológicamente la zona se encuentra 
clasificada como zonobioma tropical alternohidrico (5). Topográficamente el $80 \%$ del terreno de la hacienda Mundo Nuevo es plano e inundable en los periodos lluviosos. Registra una temperatura promedio anual de $27^{\circ} \mathrm{C}$ y una pluviosidad de 1.400 a $1.600 \mathrm{~mm}$ anuales, marcada en un periodo lluvioso, en el que caen $85 \%$ de las lluvias (mayo a octubre) y un periodo seco (noviembre a abril). La hacienda Mundo Nuevo posee una extensión de 241 Hectáreas, buena disponibilidad de agua y abundantes pastizales en los que predominan, Dichantium aristatum (angleton), Bothriocloa pertusa (colosoana) y Brachiaria spp (brachiarias).

Recolección de la información. La información que se analizó correspondió a 552 registros productivos de peso al destete de los años 1997 - 2007, proveniente de las tarjetas individuales de las vacas, con lo cual se construyó una base de datos en Microsoft Excel 2007. Los pesos al destete fueron ajustados a los 270 días por formulas similares a las utilizadas por Beef Improvement Organization (4).

Análisis estadístico. El modelo estadístico utilizado incluyó los efectos fijos: año de nacimiento, época de nacimiento, sexo del ternero, número de partos de la vaca; y el efecto aleatorio del padre. El modelo fue el siguiente:

$$
Y_{i j k l m n}=u+A_{i}+B_{j}+C_{k}+P_{l}+Z_{m}+e_{i j k l m n}
$$

Donde:

$\mathrm{Y}_{\mathrm{ijklmn}}=$ Peso ajustado al destete del ternero ( kg);

$\mathrm{u}=$ Media general del peso al destete;

$A_{i}=$ Efecto del i-ésimo año de nacimiento del ternero (1997 -2007);

$B_{j}=$ Efecto de la j-ésima época de nacimiento del ternero (Seca, Lluvia);

$\mathrm{C}_{\mathrm{k}}=$ Efecto del k-ésimo sexo del ternero (Macho, Hembra);

$\mathrm{P}_{1}=$ Efecto del I-ésimo parto de la vaca (parto 1, parto 2, parto 3, parto 4 y parto 5 o más);

$\mathrm{Z}_{\mathrm{m}}=$ Efecto aleatorio del $\mathrm{m}$-ésimo toro (33 toros);

$\mathrm{e}_{\mathrm{ijk} \mid \mathrm{mn}}=$ Residual.
El análisis de varianza para los efectos fijos y aleatorios que afectan la variable peso al destete fue realizado mediante el procedimiento GLM del paquete estadístico SAS (6). Los componentes de varianza entre y dentro de padre, con los cuales se calculó la heredabilidad, fueron estimados utilizando el procedimiento Varcomp contenido en el mismo paquete.

El sistema de evaluación desarrollado para el cálculo de las DEP's para los toros, fue el de Mejor Predictor Lineal Insesgado, utilizando el mismo modelo estadístico descrito anteriormente para el cálculo de la heredabilidad. El procesamiento de los datos se realizó a través del procedimiento MIXED de SAS (6). La predicción de los valores genéticos de los toros fue expresada como diferencia esperada de progenie (DEP's) y para cada uno de ellos se calculó la exactitud (EXA), teniendo en cuenta la siguiente fórmula:

$$
E X A=1-\sqrt{\frac{S D}{\delta^{2} t}}
$$

Donde:

$\mathrm{SD}=$ Desvío estándar de la predicción;

$\delta^{2} t=$ varianza genética entre toro

Una vez hallados los valores de DEP's y la exactitud, se seleccionaron los mejores reproductores (toros), los cuales conformaran la base de sementales que serán apareados con las vacas elite de la hacienda.

\section{RESULTADOS}

La media hallada para el peso al destete (PD) fue de $238.5 \pm 31.6 \mathrm{~kg}$. En la tabla 1, se muestra el análisis de varianza para el PD. Todas las fuentes de variación tuvieron efecto altamente significativo $(p<0.001)$, excepto la época de nacimiento.

Los componentes de varianza entre y dentro de padre fueron 96.96 y 801.34 , 
Tabla 1. Análisis de varianza para el peso al destete en terneros de raza Brahman

\begin{tabular}{|c|c|c|c|c|}
\hline FV & $\mathrm{GL}$ & Cuadrados Medios & Valor $\mathrm{F}$ & $P>F$ \\
\hline Sexo & 1 & 51171.7 & 64.39 & $0.01 * *$ \\
\hline Año de nacimiento & 10 & 2002.6 & 2.52 & $0.0058 * *$ \\
\hline Número de partos & 4 & 3667.3 & 4.61 & $0.0011 * *$ \\
\hline Época de nacimiento & 1 & 3.6 & 0.0001 & $0.9457 \mathrm{NS}$ \\
\hline Padre & 32 & 1917.4 & 2.41 & $0.0001 * *$ \\
\hline Error & 503 & 794.7 & & \\
\hline
\end{tabular}

* Efecto altamente significativo $(p<0.01) ;$ N.S Efecto no significativo.

respectivamente. El valor estimado para la heredabilidad del peso al destete fue de $0.43 \pm 0.15$.

Las DEP's y su exactitud para los toros en evaluación se muestran en la tabla 2 .

Tabla 2. Diferencias esperadas de progenie y exactitud de los toros evaluados.

\begin{tabular}{cccccccc}
\hline Padre & N DEPs(kg) & EXA & Padre & \multicolumn{2}{c}{ N } & DEPs(kg & EXA \\
\hline $761-2$ & 6 & 17.0 & 0.22 & $971-5$ & 5 & -1.9 & 0.19 \\
$529-0$ & 6 & 14.0 & 0.22 & $338-5$ & 50 & -2.0 & 0.52 \\
$130-9$ & 9 & 13.5 & 0.27 & $876-4$ & 8 & -2.4 & 0.27 \\
$737-4$ & 12 & 6.8 & 0.33 & $332-6$ & 50 & -2.5 & 0.55 \\
$181-1$ & 6 & 5.8 & 0.21 & $984-8$ & 9 & -3.4 & 0.27 \\
$320-4$ & 9 & 5.5 & 0.28 & $846-8$ & 16 & -4.0 & 0.36 \\
$243-5$ & 7 & 4.9 & 0.23 & $407-7$ & 51 & -4.9 & 0.54 \\
$4702-3$ & 32 & 4.6 & 0.41 & V87-5 & 19 & -5.1 & 0.31 \\
$151-4$ & 21 & 4.3 & 0.41 & $735-1$ & 24 & -5.1 & 0.35 \\
$666-3$ & 12 & 4.0 & 0.33 & $655-2$ & 33 & -5.1 & 0.43 \\
$413-7$ & 9 & 3.6 & 0.23 & $653-7$ & 21 & -5.7 & 0.41 \\
$286-8$ & 9 & 3.0 & 0.27 & $727-0$ & 10 & -6.0 & 0.28 \\
$440-4$ & 5 & 2.1 & 0.20 & 795 & 8 & -6.3 & 0.26 \\
$449-1$ & 7 & 2.1 & 0.25 & $593-5$ & 29 & -7.6 & 0.48 \\
$151-6$ & 18 & 1.7 & 0.38 & $952-3$ & 27 & -14.2 & 0.43 \\
$517-5$ & 7 & 1.1 & 0.22 & $965-3$ & 5 & -16.9 & 0.19 \\
$489-2$ & 12 & -0.9 & 0.31 & & & & \\
\hline
\end{tabular}

El promedio de las DEP's se encuentra en el intervalo de confianza (95\%) de -2.58 a 2.58 .

En la figura 1 , se muestran el comportamiento de las DEP's y exactitudes de los toros evaluados. En

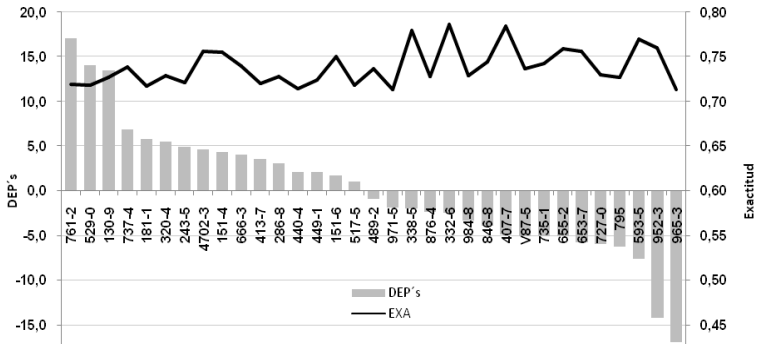

Fígura 1. DEP's y exactitudes para peso $0^{0.40} \mathrm{al}$ destete ajustado (270 días) de los toros en evaluación

la figura 2, se puede observar que en el transcurso de los años evaluados se ha venido generado una tendencia negativa del PD, presentándose una reducción promedio de $-3.3 \mathrm{~kg} / \mathrm{año}$.

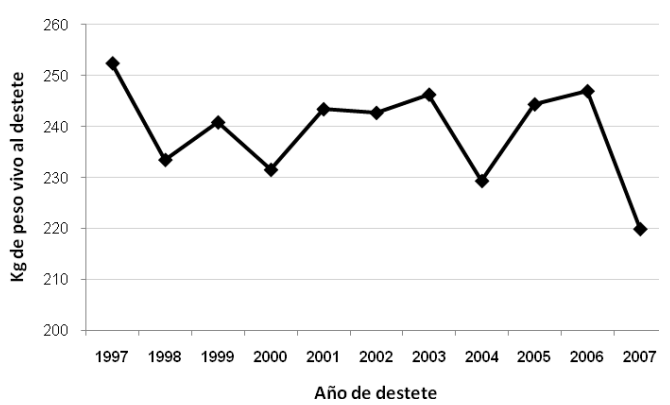

Figura 2. Tendencia fenotípica del peso al destete durante los años de evaluación

La tabla 3, presenta los 10 mejores padres de acuerdo a los valores de las DEP's.

Tabla 3. Mejores 10 padres según los valores de DEP's.

\begin{tabular}{ccccc}
\hline Padre & N & PD $(k g)$ & DEP's & EXA \\
\hline $761-2$ & 6 & 277.87 & 17.0 & 0.72 \\
$529-0$ & 6 & 264.21 & 14.0 & 0.72 \\
$130-9$ & 9 & 267.50 & 13.5 & 0.72 \\
$737-4$ & 12 & 249.35 & 6.8 & 0.74 \\
$181-1$ & 6 & 256.67 & 5.8 & 0.72 \\
$320-4$ & 9 & 245.97 & 5.5 & 0.73 \\
$243-5$ & 7 & 246.91 & 4.9 & 0.72 \\
$4702-3$ & 32 & 244.38 & 4.6 & 0.76 \\
$151-4$ & 21 & 245.30 & 4.3 & 0.76 \\
$666-3$ & 12 & 248.53 & 4.0 & 0.74 \\
\hline
\end{tabular}


En la figura 3 se puede observar la distribución de los DEP's de los toros evaluados.

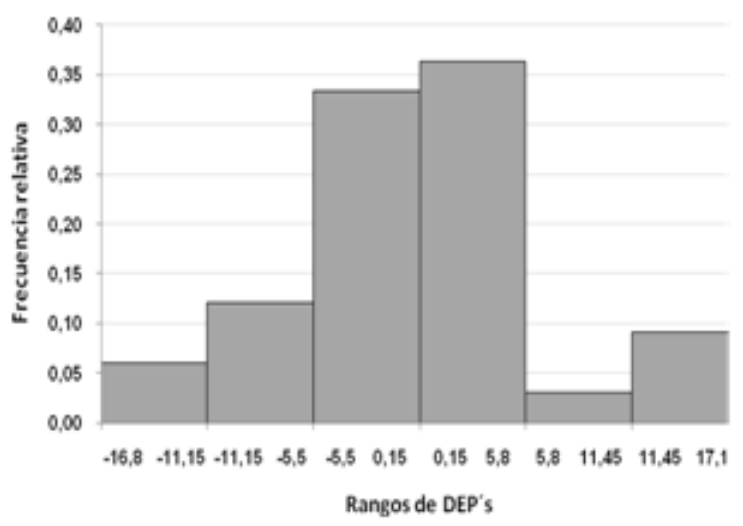

Figura 3. Histograma de frecuencias relativas de las DEP's.

\section{DISCUSIÓN}

El valor hallado para peso al destete en esta investigación es similar al encontrado por Montes et al (7) para la raza Brahman, quienes reportaron un peso ajustado a los 270 días de $237.08 \pm 35.6 \mathrm{~kg}$; valores inferiores son presentados por Quintero et al (8) y Velásquez y Álvarez (9), para la misma raza. Sin embrago, otros autores como Verde (10), Parra et al (11), Martínez-González et al (12), Atencio (13) y ASOCEBÚ (14), encontraron valores para PD ajustado a los 205 días (edad ajustada inferior a la de este estudio) para la raza Barhman de 158.6, 180.7, 185.1, 180.6 y $202 \mathrm{~kg}$, respectivamente.

El peso al destete obtenido en este estudio, se puede explicar por el plan de alimentación implementado en la finca para cubrir los periodos de escasez de forraje, las buenas prácticas de manejo animal establecida y la escogencia permanente de las novillas de reemplazo y vacas que demuestren buena habilidad materna.

El valor de heredabilidad estimado para el PD $(0.43 \pm 0.15)$ se haya dentro del rango medio, citado por Ossa et al (15). Esto sugiere que a través de la selección se puede lograr progreso genético para esta característica. La estimación de heredabilidad, hallada en esta investigación, es superior a la obtenida por Ríos (16), quien realizando una extensa revisión de literatura sobre estimaciones de parámetros genéticos para características de crecimiento predestete de bovinos encontró un promedio de heredabilidad directa para PD de 0.37. Los 504 reportes de Ríos (16) es un buen indicador de la gran variabilidad genética de esta característica. El mismo autor, realizó un promedio de estimadores de heredabilidad directa por raza, obteniendo para la raza Brahman un valor promedio de $0.26(n=17)$.

Por otro lado, autores como Verde (10) en Venezuela, Parra et al (11) en México, Quintero et al (8), Montes et al (7) en Colombia y Espinoza-Villavicencio et al (17) en México, encontraron valores de heredabilidad menores a los reportados en esta investigación $(0.10,0.33,0.29$, 0.37 y 0.05 respectivamente). Por su parte, Manrique (18) obtuvo un valor de heredabilidad similar (0.45) al hallado en el presente estudio para PD en ganado Brahman.

Martins et al (19) y Medina et al (20) registraron valores de heredabilidad para peso al destete en Nelore dentro del rango hallado en esta investigación (0.42 \pm 0.13 y $0.43 \pm 0.12$, respectivamente). No obstante, Garnero et al (21) y Gressler et al (22) presentaron valores de heredabilidad directa de 0.52 y 0.48 para la misma raza, superando el valor de 0.43 hallado en este trabajo. Otros autores, como Mañotti et al (23) y Souza et al (24), encontraron valores de heredabilidad para PD en la raza Nelore por debajo de los hallados en este trabajo.

El cálculo de la heredabilidad es de gran importancia en la predicción del valor genético de los reproductores y en la predicción de la respuesta a la selección. Por tanto, la exactitud de los estimativos del valor genético de los reproductores y de la respuesta a la selección está ligada a la precisión del estimativo de heredabilidad.

La tabla 2 muestra los valores observados de los DEP's, los cuales oscilaron entre 
-16.9 y +17.0 kg, presentando una diferencia de $33 \mathrm{~kg}$ entre el toro que menos $\mathrm{kg}$ aporta a su progenie y el que genera las crías más pesadas del hato. Barkhouse et al (25) y Cañas et al (26), reportaron para esta misma característica, estimativas de DESP's directas de 3.33 y 2.95 kg, en ganado Braman y Blanco Orejinegro, respectivamente.

En la evaluación se observa (Tabla 2), en términos generales, que el $52 \%$ de los reproductores utilizados en la hacienda aportan DEP's negativos para peso al destete, generando progenies entre -0.9 $\mathrm{kg}$ y $-16.9 \mathrm{~kg}$, por debajo del promedio de peso al destete hallado. Esto evidencia el desconocimiento del mérito genético de los reproductores usados en la finca.

DEP's altamente negativas. Corresponde a ejemplares que presentan cuyas DEP's presentan valores entre -16.9 y $-11.15 \mathrm{~kg}$. En este rango se encuentra el $6 \%$ de la población evaluada.

DEP`s medianamente negativas. En este rango se encuentran los animales cuyo peso esperado al destete para sus crías está entre -11.15 y $-5.5 \mathrm{~kg}$. Este grupo corresponde al $12 \%$ de la población de reproductores evaluada.

DEP`s negativas bajas. En esta clasificación se encuentran el 33\% de la población evaluada y presentan una diferencia esperada de progenie para PD entre -5.5 y $0,15 \mathrm{~kg}$.

DEP`s positivas bajas. Esta clasificación corresponde a los reproductores que presentan una DEP's entre 0.15 y $5.8 \mathrm{~kg}$ de PD. En este rango se encuentran el $36 \%$ de los ejemplares evaluados.

DEP `s medianamente positivas. Estegrupo corresponde al 3\% de la población evaluada y en el se encuentran los reproductores con DEP's entre 5.8 y $11.45 \mathrm{~kg}$.

DEP`s altamente positivas. Esta clasificación corresponde a los ejemplares que aportan entre 11.45 y $17 \mathrm{~kg}$ de peso vivo al destete en su progenie, los cuales representan el $9 \%$ de la población evaluada.
Partiendo de la clasificación anteriormente descrita, se observa que en la finca se ha utilizado con mayor proporción los ejemplares que aportan DEP's medianamente negativa (27\%), evidenciando que el PD puede ser mejorado en la medida en que se masifique el uso de toros que aporten DEP's positivas en el rebaño. No obstante, esta clasificación solo permite evaluar la población desde un punto de vista grupal, sin evidenciar el y/o los mejores reproductores dentro del rebaño. Por lo tanto, para comparar los reproductores dentro de la evaluación, se deben tener en cuenta, además, del valor de predicción esperado, la exactitud (EXA). Considerando que cuanto más cercano sea el valor a 1, el dato generado es más confiable y representa el verdadero merito genético del animal (27). Según Guitou (28), los valores hallados de la exactitud en esta evaluación se clasifican dentro del rango de confiabilidad alta $(0,7$ a 0,99$)$ (Tabla 2). Este valor es muy dependiente del número de registros y la calidad de los mismos, haciéndose más preciso en la medida que aumenten las observaciones por reproductor (3).

En la figura 1 se observa las diferencias esperadas de progenie y la exactitud para cada reproductor evaluado. Tomando como referencia el número de hijos presentados en la tabla 2, se observa que los reproductores identificados con los números 338-5 $(n=50), 332-6 \quad(n=50)$ y $407-7 \quad(n=51)$ presentan valores de DEP's negativos para PD (-2, -2.5 y $-4.9 \mathrm{~kg}$, respectivamente) con una exactitud que oscila entre 0.78 y 0.79 , siendo estos los más utilizados en la finca. Esta información permite evidenciar que el uso constante de reproductores que aportan DEP's negativas y un efecto de padre altamente significativo para PD, han generado una tendencia negativa en el progreso fenotípico (Figura 2) a lo largo de los años.

Como primera medida de esta evaluación genética se pueden descartar como reproductores los ejemplares anteriormente mencionados, basados en sus DEP's y EXA, los cuales indican que el mérito genético de estos ejemplares es bajo comparado con 
otros reproductores de esta evaluación, y que su valor no cambiará en el tiempo.

Partiendo de los criterios establecidos (DEP's y EXA) para seleccionar los mejores reproductores listados en la evaluación, se observa en la figura 1 , que se podrían utilizar en el programa de mejoramiento genético todos los toros con DEP's positivas, ya que traerían progreso genético para la característica de estudio. Sin embrago, los mejores resultados pueden ser obtenidos utilizando los ejemplares 761-2, 5290 y 130-9, clasificados como animales superiores dentro del 10\% (Figura 3) de la población con diferencias esperadas que generan progenies con más de $10 \mathrm{~kg}$ de peso al destete en relación al promedio del hato, y valores altos de exactitud $(>0,73)$, lo que minimiza el grado de incertidumbre en los resultados.

Evaluaciones genéticas realizadas en Colombia por ASOCEBU (14), mostraron valores de DEP's en animales superiores para PD de la raza Brahman, que oscilaron entre 10.45 y 17.21 , con EXA mínimas de 0.58. De igual manera, Ossa et al (29) evaluado la raza Romosinuano presentaron valores de $13.49 \mathrm{~kg}$ para el toro superior en la evaluación, con una exactitud de 0.92 . Sin embargo, estas referencias solo pretenden dar una idea del uso de esta herramienta en las evaluaciones genéticas y los programas de selección, dado que los toros de diferentes razas y diferentes análisis no pueden ser comparados (27).

Para interpretar los valores hallados de DEP's descritos en la tabla 2 , se deben comparar reproductores entre sí. Por ejemplo, al comparar el padre 4702-3 y el 332-6, el primero presenta un DEP's de $4.6 \mathrm{~kg}$ y el segundo $-2.5 \mathrm{~kg}$, ambos con un grado de exactitud alto, se esperaría que los hijos del primer toro presenten en promedio al destete $7.1 \mathrm{~kg}$ más que los hijos del segundo $(4.6-(-2.5)=7.1 \mathrm{~kg})$; siempre y cuando ambos toros se apareen con hembras de similar mérito genético.

Como los valores de DEP's de cada reproductor cambia con el tiempo, de acuerdo a la población base utilizada, un plan de mejoramiento genético animal en un sistema de producción, debe obedecer a un proceso sistemático de selección, hecho sobre la base de ese recurso animal. Además, no hay que perder de vista que en la medida que cambia la selección, el perfil genético de los animales también va cambiando, puesto que se va perfilando sobre aquellos que generen las mejores producciones. Sin embargo, esto puede ocasionar una pérdida de combinaciones genéticas asociadas a la resistencia y tolerancia del entorno, lo que hace necesario que la selección de animales mejorantes se realice en el lugar donde se van a desempeñar, puesto que la selección no genera nuevos genes (30).

La relación entre la respuesta y el criterio de selección se ve reflejada en el progreso genético. Teniendo como referencia la tabla 3, donde se listan los 10 mejores toros, se puede calcular el progreso genético, considerando el promedio de las DEP's de los animales seleccionados, siendo para este caso $8.04 \mathrm{~kg}$, lo cual representa el promedio de desvío estándar de las DEP's, lo que significa que la media de la población para el peso al destete en la siguiente generación tendrá un valor de $246.54 \mathrm{~kg}$, evidenciándose un aumento notable en la expresión de la característica si se programan los apareamientos con los toros seleccionados y vacas con similar merito genético.

En conclusión, el valor estimado de la heredabilidad para el peso al destete, indicaron que se puede lograr progreso genético para dicha característica.

\section{Agradecimientos}

A la Facultad de Ciencias Agropecuarias de la Universidad de Sucre, por el apoyo brindado durante todo el desarrollo de la investigación. A Inversiones Mundo Nuevo por permitir trabajar en sus instalaciones. A los colegas, Carlos Manrique y William Gómez, por toda su colaboración durante el transcurso de esta investigación. 


\section{REFERENCIAS}

1. Mahecha L, Gallego L, Peláez F. Situación actual de la ganadería de carne en Colombia y alternativas para impulsar su competitividad y sostenibilidad. Rev Col Cienc Pec 2002; 15(2):213-225.

2. Arrieta $H$, Martínez E. Estimación de parámetros genéticos para el peso al nacer en un sistema de producción en ganado doble propósito en la finca "Altamira", municipio de Ciénaga de Oro. [Trabajo de Grado]. Sincelejo, Colombia: Facultad de Ciencias Agropecuarias. Universidad de Sucre; 2001.

3. Ossa G, Suarez M, Pérez J. Valores genéticos de caracteres productivos y reproductivos en bovinos Romosinuano. Rev Corpoica 2008; 9(1).

4. Beef Improvement Federation [editorial]. Guidelines For Uniform Beef Improvement Programs. $8^{\circ}$ Edition. EEUU: The University of Georgia; 2002.

5. SAS/STAT-User's Guide. Cary, NC, USA: SAS Inst. Inc; 2006.

6. Díaz J, Gómez F. Bosque seco tropical Colombia. Bogotá: Editorial Banco de Occidente; 2006.

7. Montes D, Vergara O, Prieto E, Rodríguez A. Estimación de los parámetros genéticos para el peso al nacer y al destete en ganado bovino de la raza Brahman. Rev MVZ Córdoba 2008; 13(1):1184-1191.

8. Quintero J, Triana J, Quijano J, Arboleda E. Influencia de la inclusión del efecto materno en la estimación de parámetros genéticos del peso al destete en un hato de ganado de carne. Rev Colom Cienc Pecuaria 2007; 20(2):117-123.
9. Velásquez J, Álvarez L. Relación de medidas bovinométricas y de composición corporal in vivo con el peso de la canal en novillos Brahman en el Valle del Sinú . Rev Acta Agronómica 2004; 53(3).

10. Verde O. Sistema de Producción con Bovinos de Carne en la Estación Experimental "La Cumaca" II.- Peso a los 205 Días. Rev Fac Cs Vets 2007; 48(2):105-110.

11. Parra G, Martínez J, García F, et al. Tendencias genéticas y fenotípicas de características de crecimiento en el ganado Brahman de registro de México. Rev Científica FCV-LUZ 2007; 17(3):262-267.

12. Martínez-González J, Castillo-Rodríguez S, Lucero-Magana F, Ortega-Rivas E. Influencias ambientales y heredabilidad para características de crecimiento en ganado Sardo Negro en México. Zoot Trop 2007; 25(1):1-7.

13. Atencio A. Brahman en Venezuela pasado, presente $y$ futuro. IX seminario de pastos y forrajes y su utilización en sistemas de producción animal. [en línea] 2007 [fecha de acceso agosto 8 de 2009]. URL disponible en: http://www. avpa.ula.ve/eventos/xi_seminario/ Conferencias/Articulo-7.pdf.

14. ASOCEBU. Evaluación genética de toros Brahman 2007. [en línea] 2007 [fecha de acceso agosto 12 de 2009]. URL disponible en: http://asocebu. com/getdoc/9e27d008-fd7d-41aa9163-755fc66140ee/Evaluacion_ genetica_2007.aspx. 
15. Ossa G, Manrrique C, Torregroza L. Como utilizar los registros para evaluar animales en la finca. En: la investigación pecuaria regional 2. Cereté, Córdoba: Centro de Investigación Turipaná; 1998.

16. Ríos Á. Estimadores de parámetros genéticos para características de crecimiento predestete de bovinos. Revisión. Téc Pecu Méx 2008; 46(1):37-67.

17. Espinoza-Villavicencio $L$, PalaciosEspinosa A, Guerra-Iglesias D, González-Peña D, Ortega-Pérez R, Rodríguez-Almeida F. Comparación de dos modelos para la estimación de parámetros y valores genéticos del peso en ganado cebú. Rev Agrociencias 2008; 42(1):29-36.

18. Manrique, C. Parámetros genéticos de la raza Cebú Brahman en Colombia. Resumen. Rev Col Cienc Pec 2003; 16(4)(Supl):48.

19. Martins GA, Filho MR, Lima, Melo FAM, Lôbo RD. Influência de Fatores Genéticos e de Meio sobre o Crescimento de Bovinos da Raça Nelore no Estado do Maranhão. Rev Bras Zootec 2000; 29(1):103-107.

20. Medina J, Osorio $M$, Segura J. Influencias ambientales y parámetros genéticos para características de crecimiento en ganado Nelore en México. Revista Científica FCV-LUZ 2005; 15(3):235-241.

21. Garnero A, Gunski RJ, Schwengber EB, Lôbo RB. Comparación entre criterios de selección para características de crecimiento correlacionados con edad al primer parto en la raza nelore. Livest Rural Res Devel [en línea] 2001 [fecha de acceso Agosto 08 de 2009]; 13(2). URL disponible en: http://www.Irrd. org/Irrd13/2/garn132.htm.
22. Gressler MGM, Pereira JCC, Bergmann JAG, Andrade VJ, Paulino MF, Gressler SL. Aspectos genéticos do peso á desmama e de algumas características reprodutivas de fêmeas Nelore. Arq Bras Med Vet Zootec 2005; 57(4):533538.

23. Mañotti $A$, López $M$, Solobodzian A. Parámetros genéticos y ambientales para peso ajustado a 205 días en bovinos de la raza Nelore. Universidad Nacional del Nordeste. Rev Comunicaciones Científicas y Tecnológicas [en línea] 2003 [fecha de acceso Diciembre 12 de 2009]; 8: 1-4. URL disponible en: http://www1.unne. edu.ar/cyt/2003/comunicaciones/04Veterinarias/V-028.pdf.

24. Souza JC, Doska MC, Silva LOC, Gondo $A$, Ramos $A$, Malhado $\mathrm{CH}$, Interacción genotipo $x$ ambiente sobre el peso al destete de bovinos Nelore en Brasil. Arch Zootec 2008; 57(218):171-177.

25. Barkhouse $\mathrm{KL}$, Van Vleck LV, Cundiff L, Buchanan DS, Marshall DM. Comparison of sire breed solutions for growth traits adjusted by mean expected progeny differences to a 1993 Base. J Anim Sci 1998; 76(9):2287-2293.

26. Cañas J, Ramírez J, Arboleda O, Ochoa J, Vergara O, Cerón M. Estimación de parámetros genéticos para peso al destete en ganado Blanco Orejinegro (Bon) en el noroccidente colombiano. Rev MVZ Córdoba 2008; 13(1):11381145.

27. Plasse $D$, Arango $J$, Fossi $H$, Camaripano L, Llamozas G, Pierre A, y Romero R. Genetic and non-genetic trends for calf weights in a Bos indicus herd upgraded to pedigree Brahman. LRRD [en línea] 2004 (fecha de acceso Julio 9 de 2010); 16 (7). URL disponible en: http://www.Irrd.org/ Irrd16/7/plas16046.htm. 
28. Guitou H y Monti A. Interpretación y uso correcto de los DEPs como herramienta de selección. Unidad de Genética Animal, INTA Castelar. 1998. [fecha de acceso Agosto 12 de 2009]. URL disponible en: http://www. produccion-animal.com.ar/genetica_ seleccion_cruzamientos/deps/60interpretacion_y_uso_deps.pdf

29. Ossa G, Perez J y Suarez M. Valores genéticos de caracteres productivosy reproductivos en la raza Romosinuano. Rev Corpoica 2008; (1):93-101.
30. Ruales $F$, Manrique $C$, Cerón $M$. Fundamentos en Mejoramiento Animal. Medellín: Vieco e hijas LTDA; 2007. 